\title{
Zeno-like phenomena in STIRAP processes
}

\author{
B Militello, ${ }^{1}$ M Scala, ${ }^{1}$ A Messina, ${ }^{1}$ and $\mathrm{N} V$ Vitanov $^{2}, 3$ \\ ${ }^{1}$ Dipartimento di Scienze Fisiche ed Astronomiche dell'Università di Palermo, Via Archirafi 36, 90123 Palermo, Italy \\ ${ }^{2}$ Department of Physics, Sofia University, James Bourchier 5 blvd, 1164 Sofia, Bulgaria \\ ${ }^{3}$ Institute of Solid State Physics, Bulgarian Academy of Sciences, Tsarigradsko chaussée 72, 1784 Sofia, Bulgaria
}

\begin{abstract}
The presence of a continuous measurement quantum Zeno effect in a Stimulated Rapid Adiabatic Passage is studied, exploring in detail a sort of self-competition of the damping, which drives the system toward a loss of population and, at the same time, realizes the conditions to optimize the adiabatic passage.
\end{abstract}

PACS numbers: 03.65.Xp, 03.65.Yz, 42.50.Dv, 42.50.Lc

\section{INTRODUCTION}

Quantum Zeno effect [1] is the inhibition of the dynamics of a physical system due to repeated measurements. This occurrence has been demonstrated in different physical systems and contexts [2, 3]. In the standard treatment, measurements are assumed to be "pulsed", i.e. happening at specific instants of time, and are mathematically described by actions of projection operators. Nevertheless, in some cases the measurement can be "continuous", and in such situations a different description is required [4-8]. A very typical example is provided by a decaying level. Indeed, spontaneous decay can be thought of as an observation process, since a decay corresponds to the emission of radiation that can be revealed by a detector. Apart form interpretations, it is a matter of fact that a very strong damping is able to hinder dynamical effects [9]. More precisely, if a decaying state is coupled to another state, and if the decay rate exceeds very much the relevant coupling constant (i.e., the associated Rabi frequency), then any transition from one of these two states to the other is forbidden. This circumstance could be very helpful in some applications, where Zeno effect associated to quantum noise can compensate the detrimental effects of the noise itself. As an example, we will consider Stimulated Rapid Adiabatic Passage (STIRAP) [10], where a transfer of population from the initial state to the final one is realized through an adiabatic following [11], i.e. an adiabatic evolution [12] where an eigenstate of the Hamiltonian coincides with the initial state of the system in the initial time, and then gradually transforms into the target state. This procedure has been widely demonstrated and exploited [13-16]. Since the process involves an auxiliary state, it can be negatively affected when such auxiliary state is decaying. The effects of quantum noise have been investigated through suitable phenomenological models [17-19]. Very recently a microscopic model to describe STIRAP in the presence of environmental effects has been analyzed in details [20]. The analysis of the model proposed by Scala et al has revealed a strange behavior of the efficiency of population transfer versus the decay rate of the auxiliary state. In particular, for very small damping the effects of the environment is negligible, as expected, for stronger damping there is a visible diminishing of the efficiency, still reasonable. Instead, surprisingly, for larger values of the decay rate, the efficiency increases and reaches the maximum value, which is kept even in the limit of infinite damping. The reason of this behavior has been traced back to a dissipation-induced dynamical decoupling through qualitative arguments.

In this paper we show in detail how the presence of strong environmental effects can restore the maximum of efficiency of a STIRAP process because of the occurrence of Zeno-like phenomena. In the next section we show that a strong damping is responsible for a dynamical decoupling of the relevant state, which can preserve the population of a non-decaying state against transitions toward other states. In section III we consider the special case of STIRAP in a $\Lambda$-system in the presence of dissipation, discussing an improvement of the efficiency of STIRAP in the strong damping limit. Finally, in section IV we give some conclusive remarks.

\section{DYNAMICAL DECOUPLING INDUCED BY DISSIPATION}

It is known that a strong damping can hinder the dynamics induced by coherent couplings $[4,5,9]$. In order to better clarify this point beyond specific examples, let us consider a $N$-level system described by a time-dependent non-Hermitian Hamiltonian which takes into account possible decays $(\hbar=1)$ :

$$
H(t)=H_{s y s}(t)-i \sum_{k} \lambda \gamma_{k}(t)|k\rangle\langle k|,
$$

where $H_{\text {sys }}(t)$ is the Hermitian part of the total Hamiltonian and describes the system in the absence of dissipation, $\{|k\rangle\}$ is a basis of the Hilbert space, $\gamma_{k}(t)$ 's are the relevant decay rates, and $\lambda$ is a dimensionless parameter. Assume 
that each $\gamma_{k}$ is either identically vanishing or always strictly positive. We rewrite the Hamiltonian in the following form:

$$
H(t)=H_{0}(t)+H_{1}(t)
$$

with

$$
H_{0}(t)=\sum_{k}\left[\omega_{k}(t)-i \lambda \gamma_{k}(t)\right]|k\rangle\langle k|
$$

where $\omega_{k}(t)$ 's are the diagonal matrix elements (not necessarily the eigenvalues) of $H_{\text {sys }}(t)$.

The strong damping limit is defined by $\lambda \rightarrow \infty$. In this limit, we consider $H_{0}(t)$ as the unperturbed part and $H_{1}(t)=\left\|v_{m n}(t)\right\|$ as a perturbation. Here $v_{m n}(t)=\left\langle m\left|H_{1}(t)\right| n\right\rangle$. Once performed the transformation $|\tilde{\psi}\rangle=U(t)|\psi\rangle$, with

$$
\begin{aligned}
U(t) & =\exp \left[i \int_{0}^{t} H_{0}(s) \mathrm{d} s\right]=\sum_{k} \alpha_{k}|k\rangle\langle k|, \\
\alpha_{k}(t) & =\exp \left[\lambda \int_{0}^{t} \gamma_{k}(s) \mathrm{d} s\right],
\end{aligned}
$$

where we have used $\left[H_{0}(t), H_{0}\left(t^{\prime}\right)\right]=0$, one obtains the following Schrödinger equation:

$$
i \frac{\mathrm{d}|\tilde{\psi}\rangle}{\mathrm{d} t}=U(t) H_{1}(t) U^{-1}(t)|\tilde{\psi}\rangle .
$$

Expanding the state $|\tilde{\psi}\rangle$ in the basis $\{|k\rangle\},|\tilde{\psi}\rangle=\sum_{k} \tilde{c}_{k}|k\rangle$, one obtains the equation for the generic coefficient $\tilde{c}_{k}$, $i \mathrm{~d} \tilde{c}_{k} / \mathrm{d} t=\alpha_{k} \sum_{l} \alpha_{l}^{-1} v_{k l} \tilde{c}_{l}$, whose formal solution is

$$
\tilde{c}_{k}(t)=\tilde{c}_{k}(0)-i \int_{0}^{t} \mathrm{~d} s \sum_{l} \alpha_{k}(s) \alpha_{l}^{-1}(s) v_{k l}(s) \tilde{c}_{l}(s),
$$

by which it is possible to define the following iteration-based sequence:

$$
\tilde{c}_{k}^{(n+1)}(t)=\tilde{c}_{k}(0)-i \int_{0}^{t} \mathrm{~d} s \sum_{l} \alpha_{k}(s) \alpha_{l}^{-1}(s) v_{k l}(s) \tilde{c}_{l}^{(n)}(s) .
$$

This gives rise to the first-order (i.e., one-iteration) approximated solution:

$$
\tilde{c}_{k}^{(1)}(t)=c_{k}(0)-i \int_{0}^{t} \mathrm{~d} s \sum_{l} \alpha_{k}(s) \alpha_{l}^{-1}(s) v_{k l}(s) c_{l}(0),
$$

where we have used $\tilde{c}_{k}^{(0)}(t)=\tilde{c}_{k}(0)=c_{k}(0)$.

Therefore, the coefficients of the expansion $|\psi\rangle=\sum_{k} c_{k}|k\rangle$ are given, at first order, by the following expression:

$$
c_{k}^{(1)}(t)=\alpha_{k}^{-1}(t)\left[c_{k}(0)-i \sum_{l} \int_{0}^{t} \mathrm{~d} s \alpha_{k}(s) \alpha_{l}^{-1}(s) v_{k l}(s) c_{l}(0)\right] \text {. }
$$

Now, if $\gamma_{k}(t) \neq 0$ and $\lambda$ increases, then $c_{k}(0) \alpha_{k}^{-1}(t) \rightarrow 0$, and the second contribution in the right hand side member vanishes as well (see Appendix A). Instead, if $\gamma_{k}(t)=0$ then $c_{k}(0)$ acquires a possible time-dependent phase factor related to $\omega_{k}(t)$, while, in the $\lambda \rightarrow \infty$ limit, the only surviving terms in the summation over $l$ are those for which $\gamma_{l}(t)=0$. Summarizing, for large $\lambda$ :

$$
\begin{cases}c_{k}^{(1)}(t) \approx 0, & \text { if } \gamma_{k}(t) \neq 0 \\ c_{k}^{(1)}(t) \approx e^{-i \int_{0}^{t} \omega_{k}(s) \mathrm{d} s}\left[c_{k}(0)-i \sum_{l: \gamma_{l}(t)=0} c_{l}(0) \int_{0}^{t} e^{i \int_{0}^{s} \omega_{k l}(r) \mathrm{d} r} v_{k l}(s) \mathrm{d} s\right], & \text { if } \gamma_{k}(t)=0\end{cases}
$$

with $\omega_{k l}(t)=\omega_{k}(t)-\omega_{l}(t)$. 
On this basis we can say that there are no transitions from the decaying states toward the non-decaying ones, and vice versa. Moreover, the dynamics within the non-decaying subspace is essentially unitary, according to the firstorder relation between the coefficients and the matrix elements of the interaction Hamiltonian, $v_{k l}(s)$ 's. Therefore, the total population of the non-decaying subspace is conserved since there are not such processes like transitions to decaying states and consequent losses of population. Instead, the decaying states lose all their initial population, as one could expect. As a special case, in the strong damping limit, if there is a single non-decaying state, then the time evolution preserves the population of such state through all the process, while all the other states get empty if they are initially populated.

\section{STIRAP AND DISSIPATION}

In a recent paper [20] Scala et al have analyzed a Stimulated Rapid Adiabatic Passage in a $\Lambda$-system and in the presence of dissipation. The Hamiltonian model describing the system driven by pulses in the absence of dissipation, expressed in the basis $\{|1\rangle,|2\rangle,|3\rangle\}$, is (with $\hbar=1$ ):

$$
H_{0}(t)=\left[\begin{array}{ccc}
\omega_{1} & \Omega_{p}(t) e^{i\left(\omega_{21}-\Delta\right) t} & 0 \\
\Omega_{p}(t) e^{-i\left(\omega_{21}-\Delta\right) t} & \omega_{2} & \Omega_{s}(t) e^{-i\left(\omega_{23}-\Delta\right) t} \\
0 & \Omega_{s}(t) e^{i\left(\omega_{23}-\Delta\right) t} & \omega_{3}
\end{array}\right],
$$

where $\omega_{k}$ 's are the free energies of the three states, $\Omega_{p}(t)=\Omega_{0} f_{p}(t)$ and $\Omega_{s}(t)=\Omega_{0} f_{s}(t)$ are the edges of the two pulses coupling the state $|2\rangle$ with the states $|1\rangle$ and $|3\rangle$, respectively. Moreover, $\omega_{m n}=\omega_{m}-\omega_{n}$ and $\Delta$ is the detuning between each of the two laser frequencies and the Bohr frequency of the relevant transition.

In the interaction picture associated to the transformation $A(t)=e^{i \omega_{1} t}|1\rangle\left\langle 1\left|+e^{i\left(\omega_{2}-\Delta\right) t}\right| 2\right\rangle\left\langle 2\left|+e^{i \omega_{3} t}\right| 3\right\rangle\langle 3|$, the Hamiltonian $H_{0}(t)$ becomes:

$$
H_{s}(t)=\left[\begin{array}{ccc}
0 & \Omega_{p}(t) & 0 \\
\Omega_{p}(t) & \Delta & \Omega_{s}(t) \\
0 & \Omega_{s}(t) & 0
\end{array}\right]
$$

whose instantaneous eigenstates are [17]:

$$
\begin{aligned}
& |+\rangle=\sin \varphi \sin \theta|1\rangle+\cos \varphi|2\rangle+\sin \varphi \cos \theta|3\rangle, \\
& |0\rangle=\cos \theta|1\rangle-\sin \theta|3\rangle, \\
& |-\rangle=\cos \varphi \sin \theta|1\rangle-\sin \varphi|2\rangle+\cos \varphi \cos \theta|3\rangle,
\end{aligned}
$$

where

$$
\begin{aligned}
& \tan \theta(t)=\frac{\Omega_{p}(t)}{\Omega_{s}(t)}, \quad \tan 2 \varphi(t)=\frac{2 \Omega(t)}{\Delta}, \\
& \Omega(t)=\Omega_{0} \sqrt{f_{p}(t)^{2}+f_{s}(t)^{2}}
\end{aligned}
$$

The corresponding eigenvalues are $\omega_{+}(t)=\Omega(t) \cot \varphi(t), 0$ and $\omega_{-}(t)=-\Omega(t) \tan \varphi(t)$.

Its clear that when the pulse $\Omega_{p}(t)$ precedes the pulse $\Omega_{s}(t)$, it turns out that the angle $\theta(t)$ is $\pi / 2$ in the beginning of the experiment, say $t=0$, and equal to 0 in the end of the experiment, say $t=T$. Consequently, at $t=0$ it is $|0\rangle=|1\rangle$ and at $t=T$ it turns out to be $|0\rangle=|3\rangle$. Therefore, if the Hamiltonian changes adiabatically, the dark state $|0\rangle$ can transport population of state $|1\rangle$ to state $|3\rangle$.

In a realistic situation the auxiliary state is a decaying one, and the environmental effects can reduce the efficiency of this adiabatic passage. In the paper by Scala et al [20], the quantum noise has been taken into account through a dipolar coupling involving the auxiliary state $|2\rangle$ and an "external" (to the $\Lambda$-system) state $|4\rangle$ (whose eigenenergy $\omega_{4}$ is assumed to be quite far from the other three eigenenergies), in the presence of radiation. In this model, the free-Hamiltonian describing the environment is $H_{\mathrm{E}}=\sum_{k} \omega_{k} a_{k}^{\dagger} a_{k}$, while the system-environment interaction in the Schrödinger picture is modelled by:

$$
H_{\text {noise }}=\sum_{k} g_{k}\left(a_{k}+a_{k}^{\dagger}\right) \otimes[|2\rangle\langle 4|+| 4\rangle\langle 2|] .
$$

In Ref. [20] it has been derived the relevant master equation and an effective non-Hermitian Hamiltonian. In figure 1 it is shown the efficiency of the population transfer (i.e., the final population of 


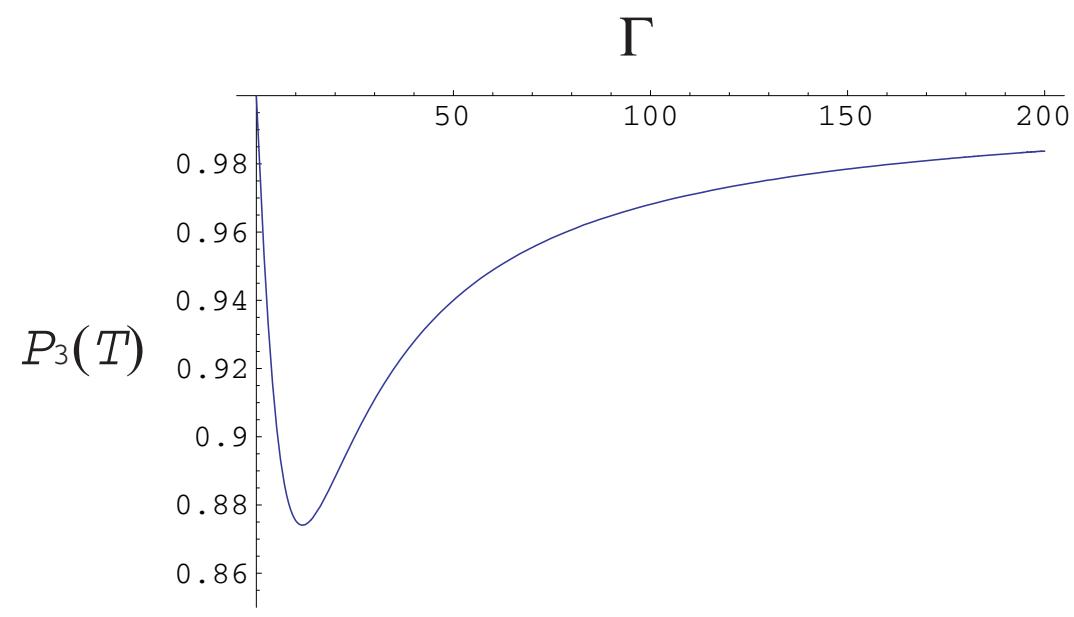

FIG. 1: The final population $P_{3}(T)$ of the state $|3\rangle$ after a time $T$ corresponding to the total duration of the experiment, as a function of the parameter $\Gamma$ (in units of $\tau^{-1}$ ). The values of the relevant parameters are such that $\Omega_{0} \tau=10, \tau \Delta=0.1$ and $T=10 \tau$, where $\tau$ represents the width of each of the two pulses. In the intermediate region there is a decrease of efficiency of the population transfer, while in the limit $\Gamma \rightarrow \infty$ the efficiency tends toward unity.

the state $|3\rangle$ after a time $T$ corresponding to the total duration of the experiment) as a function of the decay rate of state $|2\rangle$ toward $|4\rangle$. In accordance with Refs $[17,20]$, the two pulse edges are assumed to be the following: $\Omega_{p}(t)=\Omega_{0} /(\tau \sqrt{2}) \operatorname{sech}((t-T / 2) / \tau) \sin [(\tanh ((t-T / 2) / \tau)+1) \pi / 4]$ and $\Omega_{s}(t)=$ $\Omega_{0} /(\tau \sqrt{2}) \operatorname{sech}((t-T / 2) / \tau) \cos [(\tanh ((t-T / 2) / \tau)+1) \pi / 4]$. The values of the relevant parameters are $\Omega_{0} \tau=10$ and $\tau \Delta=0.1$, while the total duration of the experiment is assumed to be $T=10 \tau$. It is well visible that for small damping, i.e. $\Gamma \rightarrow 0$, the efficiency is close to unity. Then, for increasing $\Gamma$, the efficiency reasonably decreases, but, for larger values of $\Gamma$, the predicted final population of state $|3\rangle$ is again close to 1 .

The behavior of the population $P_{3}(T)$ can be explained with the results of section II. In fact, In Ref. [20] it has been proven that the effective Hamiltonian describing the dissipating system in the basis $\{|+\rangle,|0\rangle,|-\rangle\}$ is:

$$
\begin{aligned}
H_{\mathrm{eff}} & =\lambda\left[\begin{array}{ccc}
-i \gamma_{+}(t) & 0 & 0 \\
0 & 0 & 0 \\
0 & 0 & -i \gamma_{-}(t)
\end{array}\right] \\
& +\left[\begin{array}{ccc}
\Omega \cot \varphi & i \dot{\theta} \sin \varphi & i \dot{\varphi} \\
-i \dot{\theta} \sin \varphi & 0 & -i \dot{\theta} \cos \varphi \\
-i \dot{\varphi} & i \dot{\theta} \cos \varphi & -\Omega \tan \varphi
\end{array}\right]
\end{aligned}
$$

with $\lambda=\Gamma / \Omega_{0}, \gamma_{+}(t)=\Omega_{0} \cos ^{2} \varphi>0, \gamma_{0}(t)=0, \gamma_{-}(t)=\Omega_{0} \sin ^{2} \varphi>0$, and where explicit dependence of $\Omega, \theta$ and $\varphi$ on $t$ has been omitted. Now, since the state $|0\rangle$ carries the population and for $\lambda \rightarrow \infty$ the damping does not affect the dynamics of such state, then in the limit of strong damping a perfect population transfer from $|1\rangle$ toward $|3\rangle$ is restored.

It is worth commenting the presence of the minimum in the figure. In fact, its occurrence relies on the facts that $P_{3}(T) \rightarrow 1$ both for $\Gamma \rightarrow 0$ and $\Gamma \rightarrow \infty$, and that for small non-vanishing values of $\Gamma$ (when the Zeno dynamics cannot be established) the damping has only detrimental effects.

\section{DISCUSSION AND CONCLUSIVE REMARKS}

Summarizing, in this paper we have given a detailed explanation of the restoration of a high efficiency of the population transfer realized through a STIRAP process, in the presence of quantum noise. Environmental effects initially reduce the efficiency because of a certain loss of probability of the dark state that carries the population from the initial to the target one. Since the dark state is not directly decaying (the relevant diagonal matrix element of the Hamiltonian does not possess an imaginary part) this loss of probability is due to small transitions to the 
other states provoked by the off-diagonal terms of the Hamiltonian, then followed by spontaneous decay. When the damping increases, a dynamical decoupling is induced. In fact, the differences between the three diagonal terms of the Hamiltonian (the complex counterparts of the Bohr frequencies) become much larger than the off-diagonal elements, avoiding transitions. Therefore, the dynamics of the dark state is not affected neither by the interaction with the other states nor by their decay. In a sense, we can say that quantum noise competes with itself. Indeed, on the one hand it tends to provoke loss of population, while, on the other hand, a strong damping perfectly isolates the only non-decaying state, then realizing the conservation of the probability of the population carrier.

We conclude with some comments on the approximated solution in eq. (9) and the consequent behavior expressed in eq. (10). In fact, it possible to reach the same conclusion at any order. Indeed, looking at eq. (7), one sees that, whatever is the approximation order, the integrand is always the product of an exponential and a bounded function, and it is still applicable the statement proven in A. Therefore, at any order, in the $\lambda \rightarrow \infty$ limit, most of the coefficients (those corresponding to states with non-vanishing decay rates) approach zero, while the others (those corresponding to states with vanishing decay rates) are modified as if a unitary dynamics governs the relevant subspace.

\section{Acknowledgements}

This work is supported by European Commission's projects EMALI and FASTQUAST, and the Bulgarian Science Fund grants VU-F-205/06, VU-I-301/07, and D002-90/08. Support from MIUR Project N. II04C0E3F3 is also acknowledged. 


\section{Appendix A}

In this appendix we prove some statements that have been exploited through this paper.

Statement - Let $\Gamma(t)$ be a continuous monotonic increasing and nonnegative function defined in the interval $[0, T]: \Gamma(t) \geq 0 ; t>t^{\prime} \Rightarrow \Gamma(t)>\Gamma\left(t^{\prime}\right)$. Then the integral

$$
I(\lambda, t)=e^{-\lambda \Gamma(t)} \int_{0}^{t} e^{\lambda \Gamma(s)} \mathrm{d} s,
$$

has the following property:

$$
\lim _{\lambda \rightarrow \infty} I(\lambda, T)=0
$$

Proof - We want to prove that $\forall \xi>0 \quad \exists \lambda_{\xi}: \lambda>\lambda_{\xi} \Rightarrow 0<I(\lambda, T)<\xi$.

It is obvious that the quantity $I(\lambda, t)$ is always strictly positive. Moreover, For any $\eta>0$ the following occurs:

$$
\begin{aligned}
I(\lambda, T) & =e^{-\lambda \Gamma(t)} \int_{0}^{T-\eta} e^{\lambda \Gamma(s)} \mathrm{d} s+e^{-\lambda \Gamma(t)} \int_{T-\eta}^{T} e^{\lambda \Gamma(s)} \mathrm{d} s \\
& \leq(T-\eta) e^{-\lambda(\Gamma(T)-\Gamma(T-\eta))}+\eta, \\
& \leq \eta+T e^{-\lambda(\Gamma(T)-\Gamma(T-\eta))} .
\end{aligned}
$$

Now, choosing $\eta=\xi / 2$ and $\lambda_{0}=-[\Gamma(T)-\Gamma(T-\xi / 2)]^{-1} \ln (\xi / 2 T)$, for any $\lambda>\lambda_{0}$ one has

$$
0<I(\lambda, T)<\xi
$$

which proves the assertion.

On this basis, one can immediately prove the following:

Statement - Given a function $\Gamma(t)$ satisfying the hypotheses of the previous statement, and a complex and integrable function $f$ whose modulus is bounded in the interval $[0, T]$, it turns out that

$$
\lim _{\lambda \rightarrow \infty} e^{-\lambda \Gamma(T)} \int_{0}^{T} e^{\lambda \Gamma(s)} f(s) \mathrm{d} s=0 .
$$

Proof - The modulus of the limit argument is smaller than $I(\lambda, T) \times \max _{[0, T]}|f|$.

Because of the structure of the $\alpha_{k}$ 's coefficients given by eq. (4), eq. (A5) proves that the second term in eq. (9) goes to zero when $\lambda \rightarrow \infty$ and $\gamma_{k}(t)>0$. Indeed, for $\gamma_{k}(t)>0$ (state $|k\rangle$ is decaying) and $\gamma_{l}(t) \geq 0$ (state $|l\rangle$ is decaying or not) it turns out that the function $\Gamma(t)=\int_{0}^{t} \gamma_{k}(s) \mathrm{d} s$ satisfies the hypotheses of the previous statement, and that $\alpha_{l}^{-1}(s) v_{k l}(s)$ is a bounded integrable function.

[1] Misra B and Sudarshan E C G 1997 J. Math. Phys. 18, 7456.

[2] Itano W M, Heinzen D J, Bollinger J J and Wineland D J 1990 Phys. Rev. A 412295.

[3] Wilkinson S R, Bharucha C F, Fischer M C, Madison K W, Morrow P R, Qian Niu, Bala Sundaram, and Raizen M G 1997 Nature 387, 575.

[4] Schulman L S 1998 Phys. Rev. A 57, 1509.

[5] Home D and Whitaker M A B 1997 Ann. Phys. 258, 237.

[6] P. Facchi and S. Pascazio, Progress in Optics 41 edited by E. Wolf, Elsevier, Amsterdam, 2001.

[7] Facchi P and Pascazio S 2008 J. Phys. A: Math. Theor. 41, 493001.

[8] Facchi P, Marmo G, and Pascazio S 2009 J. Phys.: Conf. Ser. 196, 012017.

[9] Panov A D 1999 Phys. Lett. A 260, 441; Audretsch J, Mensky M B, Panov A D 1999 Phys.Lett. A $261,44$.

[10] For reviews see: Bergmann K, Theuer H, and Shore B W 1998 Rev. Mod. Phys. 70, 1003; Vitanov N V, Fleischhauer M, Shore B W and Bergmann K 2001 Adv. At. Mol. Opt. Phys. 46, 55; Vitanov N V, Halfmann T, Shore B W and Bergmann K 2001 Ann. Rev. Phys. Chem. 52, 763; Král P, Thanopoulos I, and Shapiro M 2007 Rev. Mod. Phys. 79, 53.

[11] Cirac J I, Blatt R, and Zoller P 1994 Phys. Rev. A, 49, R3174.

[12] Messiah, Quantum Mechanics (Dover publications, 1999).

[13] Gaubatz U, Rudecki P, Schiemann S, Bergmann K 1990 J. Chem. Phys. 92, 5363.

[14] Schiemann S, Kuhn A, Steuerwald S, Bergmann K 1993 Phys. Rev. Lett. 71, 3637. 
[15] Klein J, Beil F, and Halfmann T 2007 Phys. Rev. Lett. 99, 113003.

[16] Klein J, Beil F, and Halfmann T 2008 Phys. Rev. A 78, 033416.

[17] Vitanov N V and Stenholm S 1997 Phys. Rev. A 56, 1463.

[18] Ivanov P A, Vitanov N V, and Bergmann K 2004 Phys. Rev. A 70, 063409.

[19] Ivanov P A, Vitanov N V, and Bergmann K 2005 Phys. Rev. A 72, 053412.

[20] Scala M, Militello B, Messina A, Vitanov N V 2010 Phys. Rev. A 81, 053847. 